\title{
Indirect Exchange Interactions in Orthorhombic Lanthanide Aluminates
}

\author{
D. Petrov* AND B. AngelOV \\ University of Plovdiv, Dept. of Physical Chemistry, 24, Tsar Asen Str., 4000 Plovdiv, Bulgaria
}

(Received December 14, 2011; in final form August 14, 2012)

\begin{abstract}
Magnetic exchange interactions in orthorhombic lanthanide monoaluminates $\mathrm{LnAlO}_{3}, \mathrm{Ln}=\mathrm{Sm}-\mathrm{Yb}$ have been determined semiempirically within the Anderson model of exchange in insulators. The constants of magnetic exchange have been evaluated assuming indirect exchange between pairs of nearest $\mathrm{Ln}^{3+}-\mathrm{Ln}^{3+}$ ions via $4 f^{n} \rightarrow 4 f^{n-1} 5 d$ excitation and ionic polarization. The transfer integrals have been approximated by two-centre overlap integrals between four-term analytical $4 f^{n}$ radial wave functions of $\mathrm{Ln}^{3+}$ ions. The results have been compared with the available experimental Néel temperatures in the same compounds. It has been found that in most cases the indirect exchange interactions present substantial contributions to the energy of the antiferromagnetically ordered state in $\mathrm{LnAlO}_{3}$.
\end{abstract}

PACS: $75.30 . \mathrm{Kz}, 75.30 . \mathrm{Et}$

\section{Introduction}

The orthorhombic lanthanide monoaluminates (OLA) $\mathrm{LnAlO}_{3}(\mathrm{Ln}=\mathrm{Sm}-\mathrm{Yb})$ crystallize in the $\mathrm{GdFeO}_{3}$-type structure, space group Pbnm and comprise the dominant part in the series of lanthanide aluminium perovskites. The orthorhombic structure of $\mathrm{SmAlO}_{3}$ to $\mathrm{YbAlO}_{3}$ has been found stable between $12 \mathrm{~K}$ and $1200 \mathrm{~K}$ approximately [1]. The remaining three members of the lanthanide series with open $4 f^{n}$ subshell (excluding $\mathrm{Pm}$ ) exhibit different deviations from the perovskite structure, namely $\mathrm{CeAlO}_{3}(P 4 / m m m), \mathrm{PrAlO}_{3}(R \overline{3} m)$ and $\mathrm{NdAlO}_{3}(R \overline{3} c)$ [1]. Lanthanide monoaluminates have been noted with very good microwave dielectric properties, especially $\mathrm{SmAlO}_{3}[2,3]$; a ferroelastic transformation has been studied in the latter [4]. It has been also found that the magnetic properties of $\mathrm{LnAlO}_{3}$ affect their quality factors for applications as microwave dielectric resonators [3].

The magnetic properties of the OLA include the following features: (i) their Néel temperatures are very low: below $4.2 \mathrm{~K}$, or below $1 \mathrm{~K}$, or not measured, (ii) there exists a large magnetic anisotropy, except for $\mathrm{GdAlO}_{3}$ (iii) field induced magnetization, (iv) metamagnetism below the magnetic ordering temperature and unusually low effective magnetic moment of $\mathrm{Dy}^{3+}$ ions in nanocrystalline $\mathrm{DyAlO}_{3}[5] . \mathrm{Eu}^{3+}$ ions in $\mathrm{EuAlO}_{3}$ occupy two magnetically nonequivalent sites of monoclinic symmetry $C_{1 h}[6]$, whereas $\mathrm{DyAlO}_{3}$ contains four magnetic sublattices [7]. Comparative crystal-field analysis has been performed in [8] to study the influence of the low-symmetry site of $\mathrm{Tb}^{3+}$ in $\mathrm{TbAlO}_{3}$ on its spectroscopic and magnetic properties. It has been accepted that the low orthorhom-

\footnotetext{
* corresponding author; e-mail: petrov_d_n@abv.bg
}

bic symmetry of $\mathrm{LnAlO}_{3}$ predetermines the anisotropy of their magnetic susceptibilities $[6,7]$.

The contributions to the magnetic ordering energy have been attributed to various mechanisms and factors, including magnetic exchange interactions, magnetic dipolar interactions, and components of the $g$-tensor.

The aim of this study is to obtain the magnitude of magnetic exchange in the orthorhombic $\mathrm{LnAlO}_{3}$ on a semiempirical basis adopting an appropriate physical model.

\section{Method}

As mentioned above, the orthorhombic $\mathrm{LnAlO}_{3}$ are insulators and the Anderson model of magnetic exchange in insulators should be applicable in this case. It seems reasonable to employ the indirect mechanism of magnetic exchange since: (i) the cation-cation distances are shorter than the distances $\mathrm{Ln}-\mathrm{O}-\mathrm{Ln}$ and hence have greater importance than the superexchange links via oxygen ions, (ii) the corresponding covalency parameter $\lambda$ of each bond $\mathrm{Ln}-\mathrm{O}$ rarely exceeds $2-3 \%$. The physical model used below is based on two requirements noted by Anderson [9]: (i) the inclusion of low-lying electronic excitations which are spin oriented (e.g., upward spins in one set of ions and downward spins in the other); (ii) an insulator should not be considered diluted with ions possessing definite magnetic moments (here, $\mathrm{Ln}^{3+}$ ) - since the molar ratio $\mathrm{Ln}_{2} \mathrm{O}_{3}: \mathrm{Al}_{2} \mathrm{O}_{3}=1: 1$ in $\mathrm{LnAlO}_{3}$.

The constants of magnetic exchange interactions arising from cation-cation contributions may be determined by the expression $[9,10]$ :

$$
J_{\text {ex }}=-B_{i j}^{2} / 2 n_{r} U \text {, }
$$

where $B_{i j}$ are integrals of electron transfer between the two centres $i$ and $j$ without change of spin, $n_{r}=n_{S} / n_{f}$ is the relative number of electrons with unpaired spins 
$n_{S}$ in respect of the overall number $n_{f}$ of $4 f$ electrons of each lanthanide ion, $U$ is the electrostatic energy of the excited state through which the indirect exchange interactions proceed; $J_{\text {ex }}$ is negative for antiferromagnetic exchange. The $B_{i j}$ integrals have been approximated by $\mathrm{Ln}^{3+}-\mathrm{Ln}^{3+}$ orbital overlap,

$$
B_{i j}^{2}=\left(\langle 4 f \mid 4 f\rangle_{\sigma}+\langle 4 f \mid 4 f\rangle_{\pi}\right)^{2},
$$

where the sum of two-centre overlap integrals of $\sigma$ - and $\pi$-type are over non-repeated pairs of cations in the unit cell; the last expression also preserves the correct dimension of energy for the right-hand side in expression (1). The necessary $\mathrm{Ln}^{3+}-\mathrm{Ln}^{3+}$ distances $\bar{R}$ have been those reported by Vasylechko et al. [1].

The energy $U$ has been represented through a sum of excitation energy, $E(4 f \rightarrow 5 d)$ and polarization energy, $E_{P}$ for each lanthanide ion

$$
U=E(4 f \rightarrow 5 d)+E_{P} .
$$

The values of the energy difference $E_{f-d}$ $\left(4 f^{n}-4 f^{n-1} 5 d\right)$ of $\mathrm{Ln}^{3+}$ have been determined by Brewer [11] and reexamined by Dorenbos [12] for halogenides and chalcogenides; the latter have been used in this study. When a rigid lattice is considered in which a hole is produced by removing the cation $\mathrm{Ln}^{3+}$ from the crystal, the resulting spherical cavity has radius $r$ in a continuous medium of relative dielectric permittivity $\varepsilon_{r}$. Hence, the polarization energy $E_{P}$ may be defined by the formula [13]:

$$
E_{P}=z^{2} e^{2}\left(1-1 / \varepsilon_{r}\right) / 2 r .
$$

The effective radius $r$ of each $\mathrm{Ln}^{3+}$ ion with 12 -fold coordination and charge $Z=+3$ has been that from [14]. For $\mathrm{LnAlO}_{3}$ with $\mathrm{Ln}=\mathrm{Sm}, \mathrm{Gd}$, Dy, and Er, experimental relative dielectric permittivities $\varepsilon_{r}$ have been used [1]; the $\varepsilon_{r}$ values of the remaining $\mathrm{LnAlO}_{3}$ from the same group, $\mathrm{Ln}=\mathrm{Eu}, \mathrm{Tb}, \mathrm{Ho}$, Tm, and $\mathrm{Yb}$, have been interpolated with the aid of the known values. The ionic polarization energy should be added to the energy of $f$ - $d$ excitation [12]; this inclusion also accounts for the distinct insulating properties of OLA by means of the relative dielectric permittivity $\varepsilon_{r}$. The necessary values of all input quantities are displayed in Table I.

The Néel temperatures have been then evaluated employing an expression in the molecular field approximation [9]:

$$
T_{N}=2 J_{\text {ex }} Z J(J+1) / 3 k \text {. }
$$

The number $Z=6$ of nearest $\mathrm{Ln}^{3+}$ neighbors of a given $\mathrm{Ln}^{3+}$ ion in the unit cell is that one determined from the crystal structure analysis of OLA [1]. It is known, e.g. for $\mathrm{DyAlO}_{3}[7]$ that the magnetic interaction between two adjacent $\mathrm{Dy}^{3+}$ ions located along the $c$ axis is stronger than that between the other four $\mathrm{Dy}^{3+}$ ions, however, we have used mean $\mathrm{Ln}^{3+}-\mathrm{Ln}^{3+}$ distance $\bar{R}$ (Table I). $J$ is the quantum number of the ground level, ${ }^{2 S+1} L_{J}$ [15]; for $\mathrm{EuAlO}_{3}$ only, the next lowest-lying level ${ }^{7} F_{1}$ has been used with $J=1$, as the ground level ${ }^{7} F_{0}$ of $\mathrm{Eu}^{3+}$ is nonmagnetic $(J=0) ; k$ is the Boltzmann constant.

All two-centre overlap integrals have been computed by a program with the aid of four-term analytical $4 f^{n}$ wave functions of $\mathrm{Ln}^{3+}$ ions [16, 17]. The polynomials needed for the computation of $\langle 4 f \mid 4 f\rangle_{\sigma}$ and $\langle 4 f \mid 4 f\rangle_{\pi}$ have been derived by operator differentiation as proposed by Lofthus [18].

The magnetic structures of OLA, e.g. of $\mathrm{DyAlO}_{3}$ [7] and $\mathrm{GdAlO}_{3}[19]$, have been based on previous neutron-diffraction [20] or structural [21] studies, respectively. Thus, for a diagrammatic presentation of the possible antiferromagnetic ordering modes in $\mathrm{LnAlO}_{3}$ the last two (or four) sources should be referred.

Previous data on magnetic interactions and energy equivalents related to $\mathrm{LnAlO}_{3}$ have been converted in this work from CGS-e.m.u. system into the SI by means of the conversion formulae and numerical factors provided in Ref. [22]. Atomic units (a.u.) have been employed in order to emphasize the relationships between the formulae (1) to (4) given above, as well as to express the other units compatible with the squared sum of the two-centre overlap integrals.

\section{Results and discussion}

Table I contains the necessary quantum numbers $S$ and $J$ implicitly in the ground level designation ${ }^{2 S+1} L_{J}$ for each lanthanide ion, relative dielectric permittivities $\varepsilon_{r}$, effective ionic radii $r$, excitation energy $E_{f-d}$, polarization energy $E_{P}$, mean interionic $\mathrm{Ln}^{3+}-\mathrm{Ln}^{3+}$ distances $\bar{R}$, and squared sum $B_{i j}^{2}$ of the two-centre overlap integrals.

The orbital overlap decreases exponentially with decreasing charge in the analytical wave functions through the lanthanide series and this effect cannot be further diminished by the decrease of the cation-cation separation. Six out of nine members of OLA have experimentally determined the Néel temperatures. In general, the calculated values of $T_{N}$ are of correct order of magnitude as far as (i) they all are below $4.2 \mathrm{~K}$, (ii) the main mechanism of contribution has been assumed to be that of indirect exchange interactions.

It should be noted that the constants of magnetic exchange $J_{\text {ex }}$ either in the literature or in this study when expressed in kelvin $(\mathrm{K})$ are in fact $J_{\mathrm{ex}} / k$, with $k$ being the Boltzmann constant.

The experimental constants of magnetic exchange $J_{\text {ex }}(\exp )$ are available for certain OLA and may be compared with the theoretical ones given in Table II. The following values pertain to $-J_{\text {ex }}(\exp ) / \mathrm{K}$ : for $\mathrm{GdAlO}_{3}$, 0.066 [19], for $\mathrm{TbAlO}_{3}, 0.034$ [7], for $\mathrm{DyAlO}_{3}, 0.024$ [7]. It is seen that $J_{\text {ex }}$ (theor) from Table II are about $20-36 \%$ of $J_{\mathrm{ex}}(\exp )$. For the same three compounds $T_{N}$ (theor) is $20-70 \%$ of $T_{N}(\exp )$. This discrepancy is due to the fact that the model used here accounts only for the isotropic contributions to the exchange interactions; the molecular field theory underestimates the crystalline anisotropy [19]. 
TABLE I

Data of $\mathrm{Ln}^{3+}$ and $\mathrm{LnAlO}_{3}$ used in the evaluation of the Néel temperature of orthorhombic lanthanide monoaluminates.

\begin{tabular}{l|c|c|c|c|c|c|c}
\hline \hline $\mathrm{LnAlO}_{3}$ & $\begin{array}{c}{ }^{2 S+1} L_{J} \text { of } \mathrm{Ln}^{3+} \\
{[15]}\end{array}$ & $\begin{array}{c}E_{f-d} / \text { a.u. } \\
{[12]}\end{array}$ & $E_{p}\left(\mathrm{Ln}^{3+}\right)_{12} /$ a.u. & $\begin{array}{c}\bar{R}\left(\mathrm{Ln}^{3+}-\mathrm{Ln}^{3+}\right) \\
{[1]}\end{array}$ & $\begin{array}{c}B_{i j}^{2} / 10^{-8} \\
(\text { a.u. })^{2}\end{array}$ & $\begin{array}{c}\varepsilon_{r} \\
{[1]}\end{array}$ & $\begin{array}{c}r\left(\mathrm{Ln}^{3+}\right)_{12} / \text { a.u. } \\
{[14]}\end{array}$ \\
\hline $\mathrm{SmAlO}_{3}$ & ${ }^{6} H_{5 / 2}$ & 0.3454 & 1.7973 & 7.064 & 56.37 & 20.4 & 2.3810 \\
$\mathrm{EuAlO}_{3}$ & ${ }^{7} F_{0}$ & 0.3886 & 1.8039 & 7.064 & 30.03 & $(19.4)$ & 2.3660 \\
$\mathrm{GdAlO}_{3}$ & ${ }^{8} S_{7 / 2}$ & 0.4338 & 1.8073 & 7.045 & 17.96 & 18.4 & 2.3546 \\
$\mathrm{TbAlO}_{3}$ & ${ }^{7} F_{6}$ & 0.2848 & 1.8196 & 7.045 & 10.91 & $(18.0)$ & 2.3357 \\
$\mathrm{DyAlO}_{3}$ & ${ }^{6} H_{15 / 2}$ & 0.3390 & 1.8290 & 7.041 & 6.525 & 17.6 & 2.3206 \\
$\mathrm{HoAlO}_{3}$ & ${ }^{5} I_{8}$ & 0.3695 & 1.8356 & 7.054 & 3.373 & 17.0 & 2.3073 \\
$\mathrm{ErAlO}_{3}$ & ${ }^{4} I_{15 / 2}$ & 0.3613 & 1.8412 & 7.052 & 1.812 & 16.3 & 2.2941 \\
$\mathrm{TmAlO}_{3}$ & ${ }^{3} H_{6}$ & 0.3581 & 1.8496 & 7.007 & 1.119 & $(16.0)$ & 2.2809 \\
$\mathrm{YbAlO}_{3}$ & ${ }^{2} F_{7 / 2}$ & 0.3978 & 1.8587 & 7.001 & 0.574 & $(15.6)$ & 2.2658
\end{tabular}

$\mathrm{GdAlO}_{3}$ is a two-sublattice antiferromagnetic material [19] whereas $\mathrm{DyAlO}_{3}$ exhibits magnetic structure of four sublattices [7]. The mechanism of the model applied in this study, though microscopic, reveals a bulk effect of the magnetic exchange caused by the existing spin arrangements.

It is seen from Table II that the indirect magnetic exchange interactions give a substantial contribution to the energy of the antiferromagnetically ordered state. It should be mentioned that at the same time the range of experimental temperatures $T_{N}$ in magnitude is relatively broad or some of them are so low that they have not been measured yet. Also, the dependence on individual parameters for particular $\mathrm{Ln}^{3+}$ ion, such as the effective ionic radii, the intrinsic $S$ - and $J$-values, is resulting to a very small value through a rather delicate balance. The temperature of magnetic ordering in $\mathrm{EuAlO}_{3}$ has not been registered on decreasing the temperature down to $1.5 \mathrm{~K}[6]$.

\section{TABLE II}

Constants of magnetic exchange interactions $J_{\mathrm{ex}}$, experimental and calculated Néel temperatures of orthorhombic lanthanide monoaluminates ${ }^{a}$.

\begin{tabular}{c|c|c|c}
\hline \hline $\mathrm{LnAlO}_{3}$ & $\begin{array}{c}-J_{\text {ex }}(\text { theor) } \\
10^{-3} \mathrm{~K}\end{array}$ & $\begin{array}{c}T_{N}[\mathrm{~K}] \\
\text { (exp.) }\end{array}$ & $\begin{array}{c}T_{N}[\mathrm{~K}] \\
\text { (theor.) }\end{array}$ \\
\hline $\mathrm{SmAlO}_{3}$ & 41 & $1.30[23]$ & 1.40 \\
$\mathrm{EuAlO}_{3}$ & 22 & - & 0.17 \\
$\mathrm{GdAlO}_{3}$ & 13 & $3.87[19]$ & 2.70 \\
$\mathrm{TbAlO}_{3}$ & 11 & $3.993[24]$ & $3.90[23]$ \\
$\mathrm{DyAlO}_{3}$ & 8.6 & $3.53[7]$ & 2.85 \\
$\mathrm{HoAlO}_{3}$ & 12 & $3.92[5]$ & 2.20 \\
$\mathrm{ErAlO}_{3}$ & 4.8 & $0.16[25]$ & 3.49 \\
$\mathrm{TmAlO}_{3}$ & 4.8 & - & 1.21 \\
$\mathrm{YbAlO}_{3}$ & 5.2 & $0.78[26]$ & 0.63 \\
\hline
\end{tabular}

${ }^{a}$ The second values of the experimental $T_{N}[\mathrm{~K}]$ refer to nanocrystalline samples.
The correspondence between experimental and theoretical values for $\mathrm{HoAlO}_{3}$ is less adequate but the former is extremely low. $\mathrm{HoAlO}_{3}$ contains a non-Kramers $\mathrm{Ho}^{3+}$ ion and exhibits different type of magnetic-field dependence of its thermal conductivity compared with DyAlO $\mathrm{Al}_{3}$ and $\mathrm{ErAlO}_{3}$ containing the Kramers $\mathrm{Ln}^{3+}$ ions, $4 f^{9}$ and $4 f^{11}$, respectively [27]. The magnetic exchange interactions in single crystal of $\mathrm{Yb}^{3+}: \mathrm{HoAlO}_{3}$ have been explained similarly as being affected by the two ground singlets of $\mathrm{Ho}^{3+}$ ion with thermal depopulation of the upper-lying ones [28].

\section{Conclusions}

The mechanism of indirect exchange used here is sensitive as it reproduces definite contributions to experimental Néel temperatures varying in a wide range within the group of OLA. These results complement other analyses that have been performed on the same group of compounds or on particular lanthanide ions [29]; in addition, they pertain to single crystals as well as to powder samples with nanosize of the particles $[5,7 ; 19,24]$. The present treatment should provoke also a systematic reexamination of the magnetic transition temperatures in the group of OLA.

\section{References}

[1] L. Vasylechko, A. Senyshyn, U. Bismayer, in: Rare Earth Aluminates and Gallates Handbook on the Physics and Chemistry of Rare Earths, Vol. 39, Eds. K.A. Gschneidner, J.-C.G. Bünzli, V.K. Pecharsky, Elsevier, Amsterdam 2009, p. 113.

[2] C.L. Huang, Y.C. Chen, Mater. Res. Bull. 37, 563 (2002).

[3] S.Y. Cho, I.T. Kim, K.S. Hong, J. Mater. Res. 14, 114 (1999).

[4] S.C. Abrahams, J.L. Bernstein, J.P. Remeika, Mater. Res. Bull. 9, 1613 (1974).

[5] D. Petrov, B. Angelov, V. Lovchinov, J. Sol-Gel Sci. Tech. 58, 636 (2011). 
[6] L. Holmes, R. Sherwood, L.G. Van Uitert, Phys. Rev. 178, 576 (1969).

[7] L.M. Holmes, L.G. Van Uitert, L.L. Hecker, G.W. Hull, Phys. Rev. B 5, 138 (1972).

[8] C. Rudowicz, P. Gnutek, V.G. Brik, J. Rare Earths 27, 627 (2009).

[9] P.W. Anderson, Solid State Phys. 14, 99 (1963).

[10] J.B. Goodenough, Magnetism and the Chemical Bond, Interscience, New York 1966, Ch. 3.

[11] L. Brewer, J. Opt. Soc. Am. 61, 1666 (1971).

[12] P. Dorenbos, J. Lumin. 91, 91 (2000).

[13] E.S. Rittner, R.A. Hutnerc, F.K. Du Prè, J. Chem. Phys. 17, 198 (1949).

[14] G.P. Williams, in: Physical Properties of the Rare Earth Metals, Ed. D. Lide, CRC Press, Boca Raton 2004-2005, p. 4.

[15] W.C. Martin, L. Hagan, J. Reader, J. Sugar, J. Phys. Chem. Ref. Data 3, 771 (1974).

[16] A.J. Freeman, R.E. Watson, Phys. Rev. 127, 2058 (1962).

[17] O.J. Sovers, J. Phys. Chem. Solids 28, 1073 (1967).

[18] A. Lofthus, Mol. Phys. 5, 105 (1962).

[19] J.D. Cashion, A.H. Cooke, T.L. Thorp, M.R. Wells, Proc. R. Soc. Lond. A 318, 473 (1970)
[20] R. Bidaux, P. Mèriel, J. Phys. (Paris) 29, 220 (1968).

[21] S. Geller, V.B. Bala, Acta Crystallogr. 9, 1019 (1956).

[22] S. Hatscher, H. Schilder, H. Lueken, W. Urland, Pure Appl. Chem. 77, 497 (2005).

[23] P.A. Arsenev, L.M. Kovba, Kh.S. Bagdasarov, B.F. Dzhurinskii, A.V. Potemkin, B.I. Pokrovskii, F.M. Spiridonov, V.A. Antonov, V.V. Ilyukhin, Compounds of Rare Earth Elements. Systems with the Oxides of I-III Group Elements, Nauka, Moscow 1983 (in Russian).

[24] D. Petrov, Appl. Phys. A 104, 1237 (2011).

[25] I. Homman, M. Ocio, Acta Crystallogr. A 33, 975 (1977).

[26] G.V. Anan'eva, A.O. Ivanov, T.I. Merkulyaeva, I.V. Mochalov, Kristallographiya 23, 201 (1978).

[27] T. Numazawa, H. Kimura, K. Shimamura, T. Fukuda, J. Mater. Sci. 33, 827 (1998).

[28] P. Bonville, J.A. Hodges, P. Imbert, J. Phys. (Paris) 41, 1213 (1980).

[29] H.W. De Wijn, A.M. Van Diepen, K.H.J. Buschow, Phys. Status Solidi 76, 11 (1976). 